\title{
NUMERICAL SIMULATION OF INCOMPRESSIBLE FLOWS AND ANALYSIS OF THE SOLUTIONS
}

\author{
Charles-Henri Bruneau ${ }^{1}$
}

\begin{abstract}
The aim of this survey is to discuss some of the difficulties one can encounter both when solving Navier-Stokes equations for incompressible flows by an obstacle and analysing the approximate solutions. Far to be exhaustive, some main aspects of the numerical simulation are deliberately pointed out, in addition to the way the obstacle is taken into account and to the far field boundary conditions. Then, using one of the robust methods it is possible to simulate the transition to turbulence for increasing Reynolds numbers. That means to compute transient solutions which need to be analyzed and here is the second topic of this paper. Indeed, the classical tools like Fourier analysis are very efficient as long as the solution is periodic but useless when the solution is more complex. Despite the development of wavelets and new algorithms it seems still difficult to distinguish quasiperiodic and chaotic solutions.
\end{abstract}

\section{Introduction}

It is nowadays quite impossible to review all the ways the researchers have found out all around the world and for thirty years to solve the Navier-Stokes equations for in- compressible flows. There are now classical books devoted to these equations and their approximations $[6,11,12,18,25,30,31]$. There are also international conferences focusing globally or partially on this topic $[17,29,23,9,16]$. This shows the success of Navier-Stokes equations among the computational fluid dynamics community. Success that gives rise to a tremendous research activity and to so many papers the reader is overwhelmed. Therefore this paper does not pretend to give an exhaustive review of the field but only some comments on some aspects of the formulations, the boundary conditions, the approximations, the solving methods and also the analysis of the solutions. Indeed, using a method robust enough on a fine mesh at least in the boundary layer area, it is now possible to compute transient solutions quite easily in $2 \mathrm{D}$ and even in $3 \mathrm{D}$ when making the best of the new computers and the new computational techniques. That means that one has to use appropriate tools of analysis to qualify the computed solutions. As long as the solutions are periodic this is very easy by Fourier analysis but when they are more complex it is very difficult to analyze precisely the solution even in laminar cases.

\footnotetext{
${ }^{1}$ Mathématiques Appliquées de Bordeaux, Université Bordeaux 1 351 cours de la Libération, 33405 Talence cedex, France.

bruneau@math.u-bordeaux.fr
} 


\section{Navier-Stokes models}

\section{$2.1 \quad$ The equations}

From the mass and momentum conservation laws, it is easy to derive Navier-Stokes equations for an incompressible Newtonian viscous fluid in a domain $\Omega \subset \mathbb{R}^{N}$ with $N \leq 3$

$$
\begin{gathered}
\partial_{t} U+(U \cdot \nabla) U-\frac{1}{R e} \Delta U+\nabla p=F \\
\text { in } \Omega_{T}=\Omega \times(0, T) \\
\operatorname{div} U=0 \text { in } \Omega_{T} .
\end{gathered}
$$

The first equation can be rewritten as

$$
\partial_{t} U+(U \cdot \nabla) U-\operatorname{div} \tilde{\sigma}(U, p)=F \text { in } \Omega_{T}
$$

or

$$
\partial_{t} U+(U \cdot \nabla) U-\operatorname{div} \sigma(U, p)=F \text { in } \Omega_{T}
$$

where $\tilde{\sigma}(U, p)$ and $\sigma(U, p)$ are respectively the pseudostress tensor and the stress tensor defined by :

$$
\begin{gathered}
\tilde{\sigma}(U, p)=\frac{1}{R e} \nabla U-p I \\
\sigma(U, p)=\frac{2}{R e} D(U)-p I \\
\text { with } D(U)_{i j}=\frac{1}{2}\left(\frac{\partial u_{i}}{\partial x_{j}}+\frac{\partial u_{j}}{\partial x_{i}}\right)
\end{gathered}
$$

with $U=\left(u_{i}\right)_{i}$ the velocity vector, $p$ the pressure, $R e$ the dimensionless Reynolds number and $F$ the external forces. Most often $F=0$ and the motion is given through a non homogeneous Dirichlet boundary condition imposed on a part $\Gamma_{D}$ of the boundary $\partial \Omega$.
These equations for the primitive variables can be transformed by introducing the vorticity $\omega=\nabla \wedge U$. The general form in $3 \mathrm{D}$ of the velocity-vorticity equations is

$$
\begin{aligned}
& \partial_{t} \omega+(U \cdot \nabla) \omega-\frac{1}{R e} \Delta \omega=(\omega \cdot \nabla) U \\
& +\nabla \wedge F \text { in } \Omega_{T}
\end{aligned}
$$

$$
\begin{aligned}
& \nabla \wedge U=\omega \text { in } \Omega_{T} \\
& \operatorname{div} U=0 \text { in } \Omega_{T}
\end{aligned}
$$

where the second part of the non linear term is treated as a source term in the first equation. In $2 \mathrm{D}$ this term vanishes. There are other models like the stream functionvorticity model which is valid only in $2 \mathrm{D}$ (see [14] for more details).

\subsection{The initial datum}

The evolution problem (2.1) (2.2) requires an initial condition

$$
U(x, 0)=U_{0}(x) \text { in } \Omega
$$

and from a mathematical point of view this initial datum must belong to the right space, in particular $U_{0}$ must a priori satisfy both the divergence-free condition and the boundary conditions. In practice it is not so easy to check the divergence-free condition and the numerical experience shows it is not compulsory. The first time steps will produce the good initial solution.

An other question related to the initial condition is the use of a high-order scheme in time requiring several initial data $U^{-j}(x), 0 \leq j \leq J$ for the first time steps. This can be solved either by setting $U^{-j}(x)=U_{0}(x)$ for $0 \leq j \leq J$ or by using an Euler scheme for these first time steps. 


\subsection{The boundary conditions}

It is well-known that the boundary conditions constitute one of the main difficulties we can encounter. Here there are three types : inflow, no-slip and outflow or open boundary conditions. The first two correspond to non homogeneous and homogeneous Dirichlet conditions, the last one is much more difficult to find out in order to get a well-posed problem and a realistic approximate solution. These conditions are gathered for instance when computing the flow behind an obstacle in a channel (figure 1) where the domain $\Omega$ has for boundary $\partial \Omega=\Gamma_{D} \cup \Gamma_{0} \cup \Gamma_{1} \cup \Gamma_{N}$.

On $\Gamma_{D}$ the flow at infinity $\left(U_{\infty}, p_{\infty}\right)$ is imposed, that is a Poiseuille flow is set at the entrance section. On $\Gamma_{1}$ there is a noslip condition $U=0$ as well as on $\Gamma_{0}$ if the mesh is adapted to the limit of the obstacle. We shall see in the next section that there are other ways to take into account the obstacle. But the condition to set on $\Gamma_{N}$ is far to be so easy. Indeed if $\Gamma_{D}$ is not too close to the obstacle $\Omega_{0}$, the Dirichlet condition is relevant at the entrance section and does not produce any perturbation. On the contrary, even when $\Gamma_{N}$ is not so close to $\Omega_{0}$ some boundary conditions can produce strong reflections when vortices are convected through the artificial limit. The treatment of the open boundary conditions for Navier-Stokes equations is itself a large field of research as nothing tell us what to do to get on the truncated domain the restriction of the solution on the infinite domain. There are essentially two ways of dealing with this difficulty which are either to use a buffer region outside of $\Omega$ in wich the equations are modified $[8,27]$ or to impose the best condition known on $\Gamma_{N}$. Many researchers have find out good open boundary conditions and we refer to [28] and references therein for more details. One of the most used is probably the zerostress boundary condition $\sigma(U, p) n=0$ we generalize in [3] by

$$
\tilde{\sigma}(U, p) n=\tilde{\sigma}\left(U_{\infty}, p_{\infty}\right) n
$$

or

$$
\sigma(U, p) n=\sigma\left(U_{\infty}, p_{\infty}\right) n
$$

for Stokes flow and by for instance

$$
\begin{aligned}
\tilde{\sigma}(U, p) n+\frac{1}{2}(U \cdot n)^{-} & \left(U-U_{\infty}\right) \\
= & \tilde{\sigma}\left(U_{\infty}, p_{\infty}\right) n
\end{aligned}
$$

or

$$
\begin{aligned}
\sigma(U, p) n+\frac{1}{2}(U \cdot n)^{-} & \left(U-U_{\infty}\right) \\
& =\sigma\left(U_{\infty}, p_{\infty}\right) n
\end{aligned}
$$

for Navier-Stokes flows with the notation $a=a^{+}-a^{-}$. On $\Gamma_{N}$, the new term is equal to zero except if $U . n$ is negative to ease the convection of vortices and avoid reflections. From a mixed formulation we can show by energy estimates that conditions (2.9) or (2.10) yield a well-posed problem [2].

\subsection{The obstacle}

To take into account the obstacle there are essentially two ways, either the mesh is constructed so that $\Gamma_{0}$ is approximated by the sides of some cells or an immersion method is used. In the first case a no-slip boundary condition $U=0$ is imposed on $\Gamma_{0}$ and the computation is done on the unstructured mesh via a finite elements or a finite volumes approximation. In the second case a cartesian mesh is applied on $D=\Omega \cup \Gamma_{0} \cup \Omega_{0}$ and the approximation is achieved by means of finite differences or spectral methods. Of 


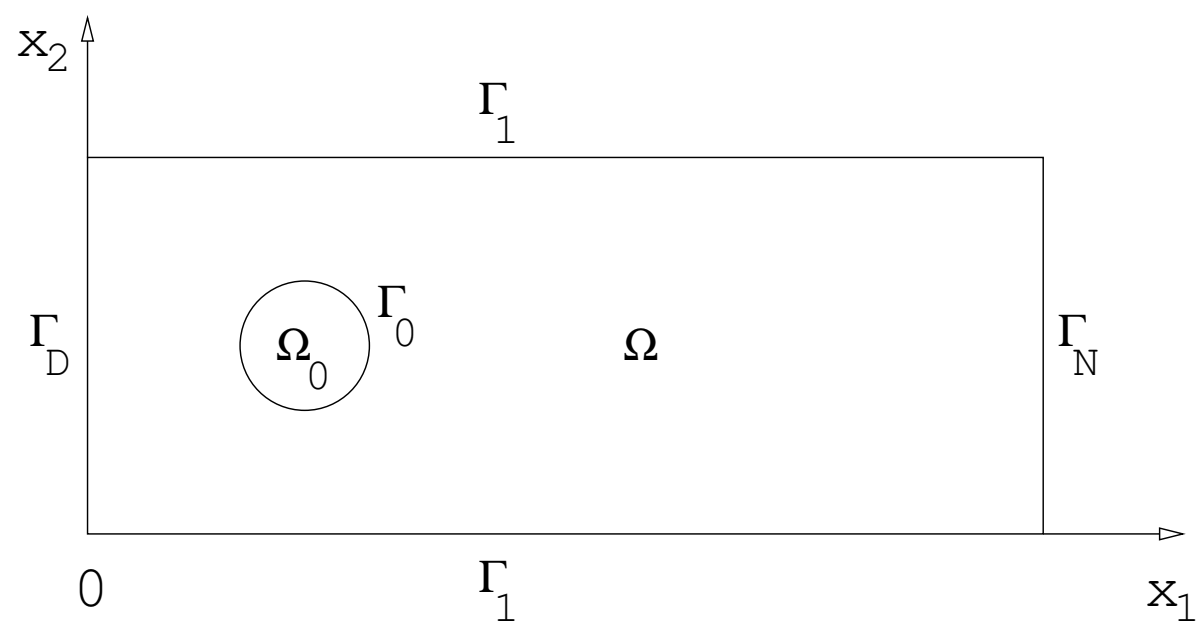

Figure 1: Computing domain

course, this needs an additional tool to represent the obstacle. One is to force the noslip condition at the surface of the body by adding a feedback forcing function to the momentun equations. The points defining the surface are chosen by the user, they can be either the closest vertices or the intersection points between the body surface and the mesh. In this second case several interpolations can be used [13, 27]. Another tool is to consider the obstacle as a porous medium with a very small permeability coefficient $K$. This yields a fluidsolid formulation in the domain $D$ by solving Navier-Stokes equations in the fluid and Darcy equations in the solid. One way to do that is to set $K=1$ at every point in $\Omega$ and to set $K=K_{\Omega_{0}} \ll 1$ at every point in $\Omega_{0}$. Then equations (2.1),(2.2) are replaced by

$$
\begin{gathered}
\partial_{t} U+(U \cdot \nabla) U-\frac{1}{R e} \Delta U+\frac{U}{R e D_{a} K^{3}} \\
+\frac{1}{K} \nabla p=F \text { in } D_{T}=D \times(0, T) \\
\operatorname{div} U=0 \text { in } D_{T}
\end{gathered}
$$

where Darcy number is given by $D_{a}=\frac{1}{R e K_{\Omega_{0}}}$. It is clear that this adds an extra work as it requires to solve equations $(2.11),(2.12)$ in $\Omega_{0}$. But the cartesian mesh simplifies the computation and allows to use the spectral or multigrid methods. Moreover, the pressure in $\Omega_{0}$ permits to compute the drag and lift forces [5]. Finally the velocity in $\Omega_{0}$ is of the same order than $K_{\Omega_{0}}$.

\section{Numerical simulation}

\subsection{The approximation}

It is obvious that we can not give here even an outline of the numerous types of approximations used to solve the Navier-Stokes equations. Indeed, this needs several books $[6,11,12,18,25,30,31]$. But, we can give a taste of the main difficulties which lay on one hand on the equilibrium between the convection and the diffusion terms and on the other hand on the divergence-free condition. It is now clear that we have to treat 
the convection term explicitely to avoid artificial numerical diffusion in time. Let us say that this term must be expressed at time $n \delta t$ when computing the solution at time $(n+1) \delta t$. On the contrary the other terms can be discretized implicitely at time $(n+1) \delta t$. The discretization in space is subjected to the mesh and thus to the way the obstacle is taken into account. The more used is probably the finite volumes approximation on unstructured meshes [24]. But with one of the immersion procedures it is possible to benefit of the spectral or finite differences methods $([19,27]$ or [4]). As the convection term is put in the second member of the momentum equation (2.1) or (2.11), a centered discretisation of the other terms yields a well-conditioned matrix easy to invert.

But the convection term needs some more work. A good discretization is needed to guarantee the success of the simulation. Indeed, one has to be very careful dealing with this term as every extra diffusion brought up by the scheme is added to $-\frac{1}{R e} \Delta U$ and changes the real value of the Reynolds number. For instance, the discretization of

$$
u \frac{\partial u}{\partial x}
$$

at point $j$ in one dimension by a first-order upwind scheme

$$
u_{j}^{n}\left(u_{j}^{n}-u_{j-1}^{n}\right) / \delta x
$$

if $u_{j}^{n}$ is positive corresponds to a secondorder approximation of

$$
u \frac{\partial u}{\partial x}-\frac{\delta x}{2} \frac{\partial}{\partial x}\left(|u| \frac{\partial u}{\partial x}\right)
$$

and thus adds a viscosity term that alters the Reynolds number. Consequently the simulation can be qualitatively correct but not quantitatively. It is well-known that the critical Reynolds number corresponding to the first Hopf bifurcation for the driven cavity problem is not yet determined for sure. Because, for this problem, this first bifurcation occurs at high Reynolds number and therefore it is not easy to achieve a good accuracy. Then it is necessary to use a less diffusive scheme ; a possible choice is to replace (3.1) by

$$
\begin{aligned}
& u_{j-1 / 2}^{n}\left(4 u_{j}^{n}-5 u_{j-1}^{n}+u_{j-2}^{n}\right) / 3 \delta x \\
& -u_{j+1 / 2}^{n}\left(4 u_{j}^{n}-5 u_{j+1}^{n}+u_{j+2}^{n}\right) / 3 \delta x
\end{aligned}
$$

if $u_{j-1 / 2}^{n}$ is positive and $u_{j+1 / 2}^{n}$ is negative. The results presented in this paper are obtained with such a scheme. For the driven cavity problem, it yields the hopf bifurcation for $R e$ around 7500 which appears in recent work to be a good value of the critical Reynolds number for this problem [23, 16]. Other results are generally obtained with high order compact schemes.

Another key to the success is the approximation of the divergence-free condition and here again there are numerous methods to do it. For a finite elements approximation, many ways were developped to find a good approximate space and often the equation (2.2) is satisfied only in a weak sense on each element [30]. With finite differences, a easy way to approximate (2.2) is to use a centered discretization on a staggered grid (figure 2) so that $\operatorname{div} U=0$ can be written at the pressure point directly without interpolation [4].

But probably the most famous way to force the incompressibility is given by the duality method as described in the next subsection.

\subsection{The convergence methods}

The last point is to find the whole method of resolution which insures a good performance 


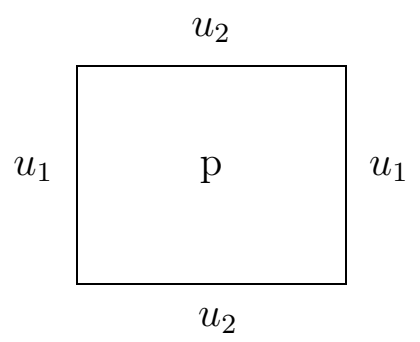

Figure 2: A staggered cell

necessary to observe the long time behaviour of the solution. As already pointed out, the duality method is one choice. Then, the pressure plays the role of the Lagrange multiplier and is computed by Uzawa's algorithm. Coupling this to a good gradient type method to invert the linear system a good performance can be achieved.

Another choice is to use the decomposition of the solution in its different scales. The new and now well-known nonlinear Galerkin method consists in cutting these scales into two parts, the large and the small ones. Then, the original NavierStokes equations are splitted into two parts to better represent the relative behaviour of the two types of scale. The result is an improvement of the performance obtained with a classical method whatever the approximation is [21]. Linked also to the different scales, the multigrid method is a very strong tool $[1,15]$. Indeed, by using successive grids it is possible to capture very fast the scales related to each grid. On one hand the solution is computed on a really coarse mesh to get the large scales and on the other hand the finer the grid is the smaller the scales can be reached $[4,33]$.

This choice of method is decisive. Indeed to make a direct simulation of the transition to turbulence it is necessary both to use a very fine mesh in the boundary layer and to compute the solution for a long time. Even in 2D, this can require several days of computing time on the best work stations.

\section{Numerical tests and analysis of solutions}

The numerical tests presented here correspond to the domain $\Omega$ of figure 1 . The channel is the rectangle $(0, L) \times(0,1)$ with $L=3$ or 4 and the obstacle is a circle of radius 0.2 which center is located at point $(1,0.5)$. We recall $R e$ is a dimensionless Reynolds number. To get a meaningful Reynolds number, one has to multiply $R e$ by the diameter of $\Omega_{0}$ which is here $d=$ 0.4. So a solution at $R e=100$ corresponds to a solution at real Reynolds number 40 . For low Reynolds numbers the numerical experiments are performed on a uniform grid of $256 \times 64$ cells which is fine enough to describe the solution. For instance, at $R e=100$ there is a symmetric steady solution with a recirculating bubble behind the cylinder as we can see on the stream function isolines (figure 3).

Then, as Re increases, the steady solution looses its stability to the benefit of a purely periodic solution very stable for higher values of $R e$. The recirculation zone alternates from the top to the bottom of the 


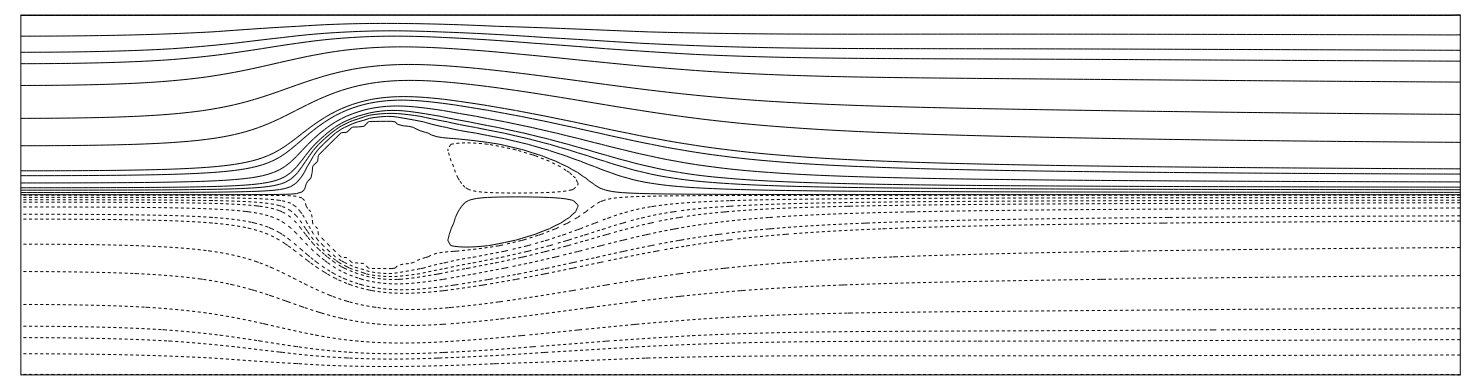

Figure 3: Solution at $R e=100$

cylinder. We can detect quite accurately the critical Reynolds number corresponding to the first Hopf bifurcation. Indeed, for this simple geometry it corresponds to the loss of symmetry. The question is : Are we sure it is quantitatively correct? To answer this question we can make another numerical test on the same geometry with $d=$ 0.2 by applying an open boundary condition on $\Gamma_{1}$ instead of the no-slip condition. In this case a constant flow $U=(1,0)$ is imposed on $\Gamma_{D}$ instead of the Poiseuille flow of flowrate 1 .

We then have a very well-known physical test and can compare the results with physical experiments. The values of the Strouhal number $S_{t}$ for various real Reynolds numbers are in very good agreement with the physics [32] and assert the accuracy of the method (table 1). Nevertheless this comparison is possible only for low Reynolds numbers.

Coming back to the initial problem and using a finer mesh, we increase $R e$ to reach other regimes. For $R e=1000$, for instance, there is still a periodic solution but this time there are strong alternate vortices convected through the domain. We can then control that the open conditions (2.10) do not affect the solution computed on a shorter domain as it can be seen on the isolines of the vorticity field (figure 4). Indeed both solutions are computed with exactly the same parameters. The only difference is the length of the domain $L=4$ and $L=3$. We see in particular that there is no reflections induced by the artificial boundary and that the computed solution on the shorter domain corresponds to the restriction of the solution computed on the larger domain at the same time (figure 4).

Then, increasing $R e$, there is still a periodic solution until $R e=3700$ but with various behaviours. Indeed, a classical Fourier analysis reveals that approximately from $R e=200$ to $R e=3700$ the flow is periodic and exhibits the same main frequency $f_{m} \simeq 1$ (this value depends on the various parameters). But from $R e=2200$ to $R e=3600$ it appears two subharmonics corresponding to $f \simeq \frac{f_{m}}{3}$ and $\frac{2 f_{m}}{3}$ and for $R e=3700$ it appears seven subharmonics corresponding to $f \simeq \frac{f_{m}}{8}$ and its multiples (figure 5). Let us note that this qualitative behaviour, in particular the number of subharmonics, changes with the geometry of the obstacle. For instance, a square of side length 0.4 does not give the same subharmonics.

Until now, the Fourier analysis is a very efficient tool that gives very accurately the frequencies of a time signal corresponding to the value of one component of the velocity at a chosen point of the domain behind the 


\begin{tabular}{|c|c|c|c|}
\hline$R e$ & Reynolds number & Computed $S_{t}$ & Value of $S_{t}$ in $[32]$ \\
\hline 300 & 60 & 0.130 & 0.136 \\
\hline 500 & 100 & 0.164 & 0.164 \\
\hline 800 & 160 & 0.188 & 0.186 \\
\hline
\end{tabular}

Table 1: Comparison of the Strouhal number
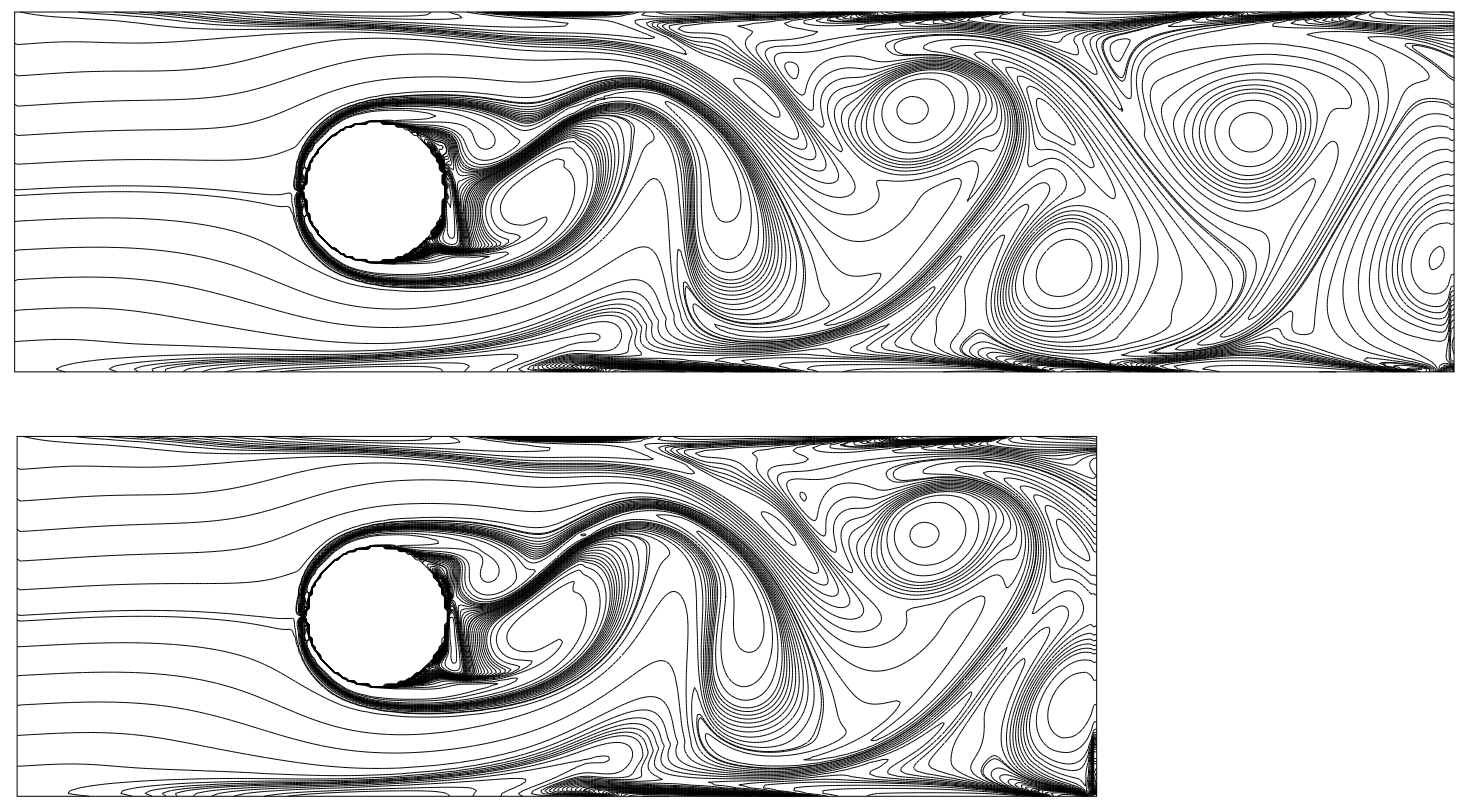

Figure 4: Comparison of the solutions obtained on the domain $\Omega$ with $L=4$ and $L=3$

cylinder. Of course, the behaviour of the solution and thus the spectrum does not depend of the point. We can complete the analysis with a phase portrait that corroborates the presence of subharmonics as the same curve is drawn several times. We see on figure 6 that a wavelets analysis is not so accurate even if the main frequency $f_{m}$ and its subharmonics are detected at $R e=3700$.

At $R e=3800$, a long time simulation shows us the solution alternates between two states. It is well-known that the wavelets are very efficient to detect a dis- continuity $[7,22,26]$ but here there is a slow transition between the two states much more difficult to analyze as it contains a large part of the spectrum. On figure 7 are represented the time-frequency analysis obtained with both a windowed Fourier transform and an adapted wavelets transform. We see the two methods provide about the same informations but as soon as there is a transition the analysis is spoiled.

When the Reynolds number increases we get more complex solutions. We can see the field becomes more complex and looks chaotic (figure 8). Nevertheless the different 

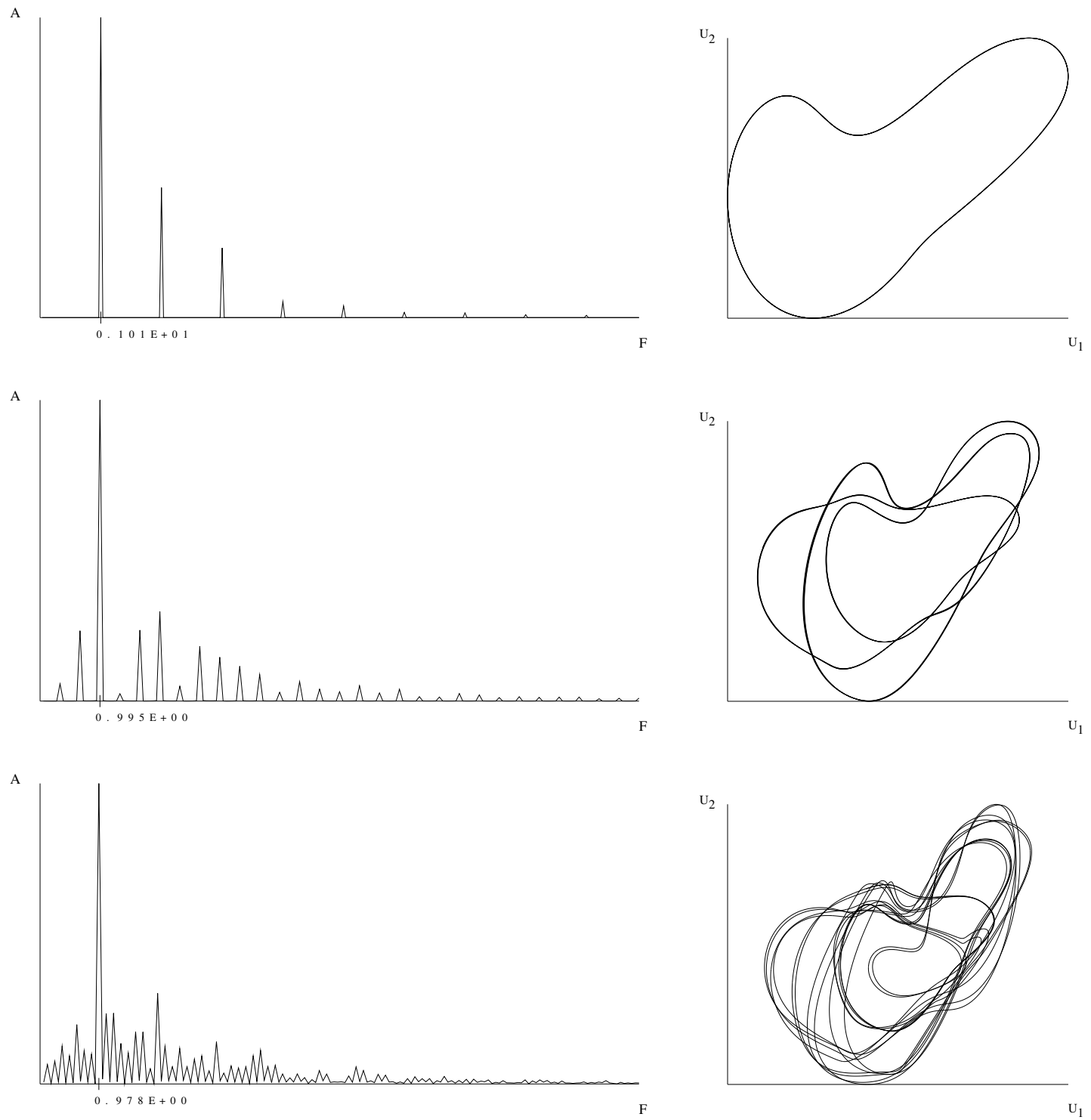

Figure 5: Spectrum and phase portrait for $R e=1000,2500$ and 3700

analysis give very few informations except the presence of the main frequency which changes with time. We think that a matching pursuit procedure [20] can give some more informations providing a good dictionary adapted to the kind of signal we analyze is used.

Another question is to understand the behaviour of the vortices, how they are convected through the domain for high Reynolds numbers, if they can merge or be divided into two parts? Here again, some work has been done by means of wavelets essentially to compress two-dimensional turbulent flows [10]. The result shows that a 2D field can be analyzed with this tool and well represented. It is now necessary to see if such an analysis from time to time can 


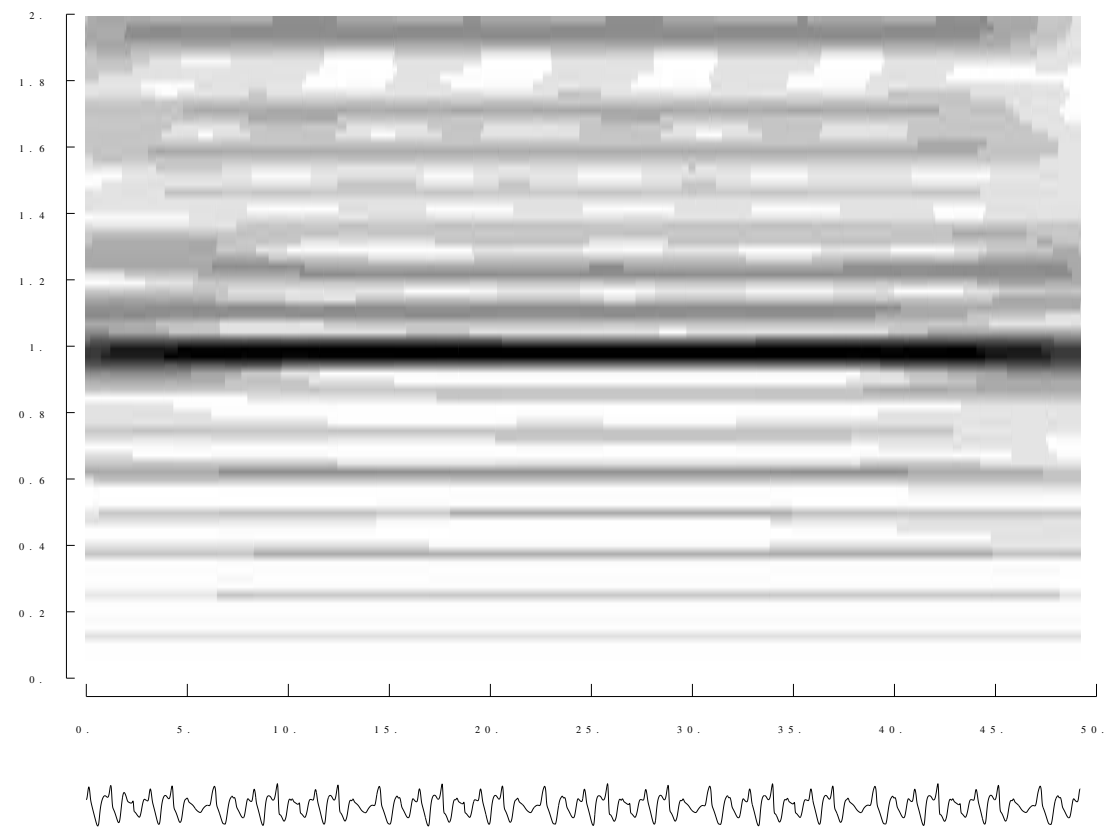

Figure 6: Wavelets analysis of the signal at $R e=3700$

give enough informations to understand the behaviour of the whole field.

\section{Conclusion}

With the improvement of both the numerical techniques and the computing power it is now possible to compute directly from the Navier-Stokes equations good transient solutions in route to turbulence. However it is still difficult to qualify the computed solutions with the existing tools of analysis.

\section{Acknowledgements}

We would like to thank Roger Gay, Bruno Torrésani and Jean-François Delage for fruitful discussions and the numerical tests on wavelets analysis.

\section{References}

[1] A. Brandt, Multigrid techniques : Guide with applications to fluid $d y$ namics, GMD-Studien 85 (1984).

[2] C.-H. Bruneau, P. Fabrie, New efficient boundary conditions for incompressible Navier-Stokes equations : A well-posedness result, $M^{2} \mathrm{AN} 30$ (1996).

[3] C.-H. Bruneau, P. Fabrie, Effective downstream boundary conditions for incompressible Navier-Stokes equations, Int. J. Num. Meth. Fluids 19 (1994).

[4] C.-H. Bruneau, C. Jouron, An efficient scheme for solving steady incom- 


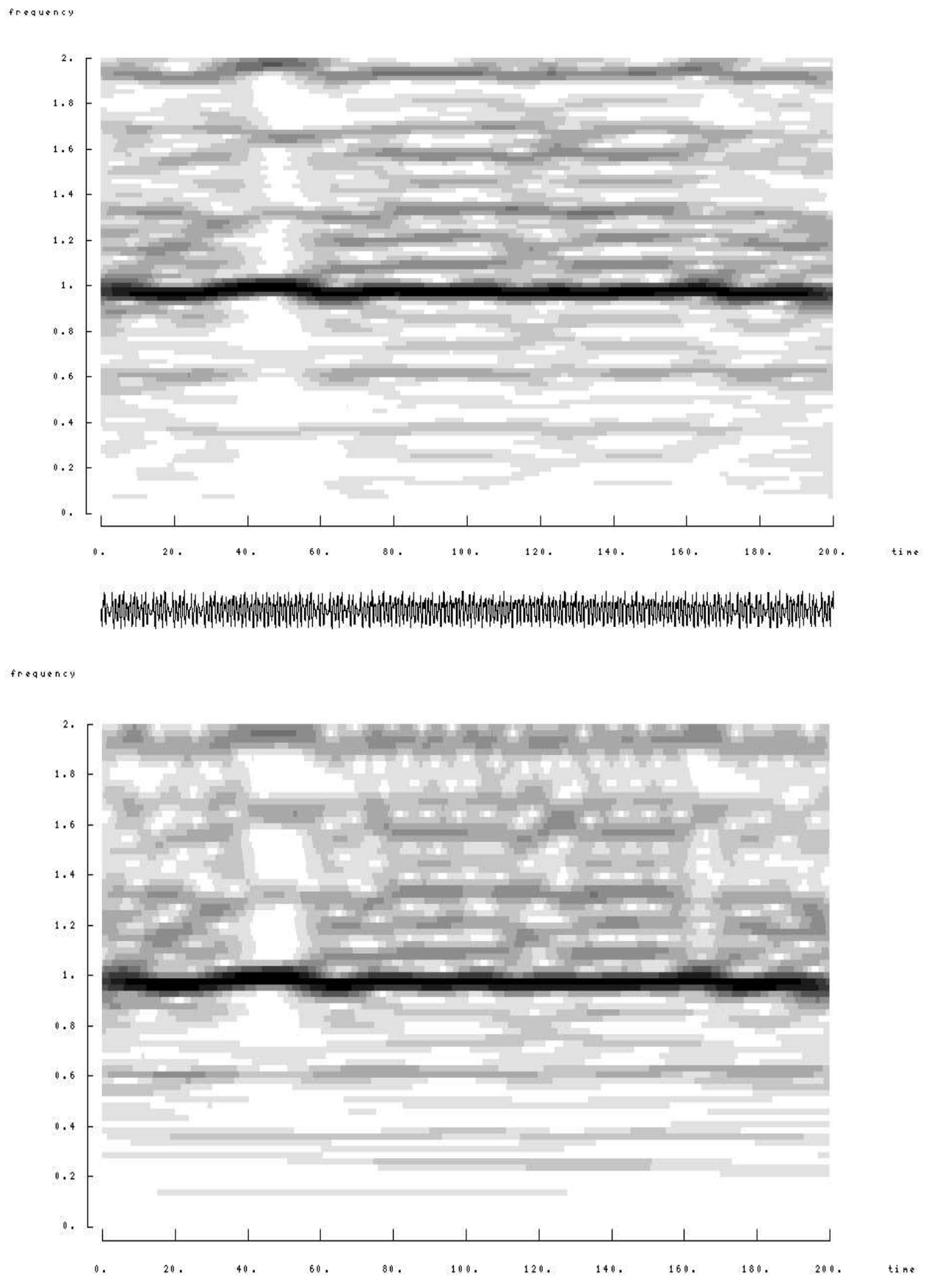

Figure 7: Windowed Fourier and wavelets analysis of the signal at $R e=3800$

pressible Navier-Stokes equations, J. Comp. Phys. 89, $n^{0} 2$ (1990).

[5] J.-P. Caltagirone, Sur l'interaction fluide-milieu poreux : Application au calcul des efforts exercés sur un obstacle par un fluide visqueux, CRAS 318, 


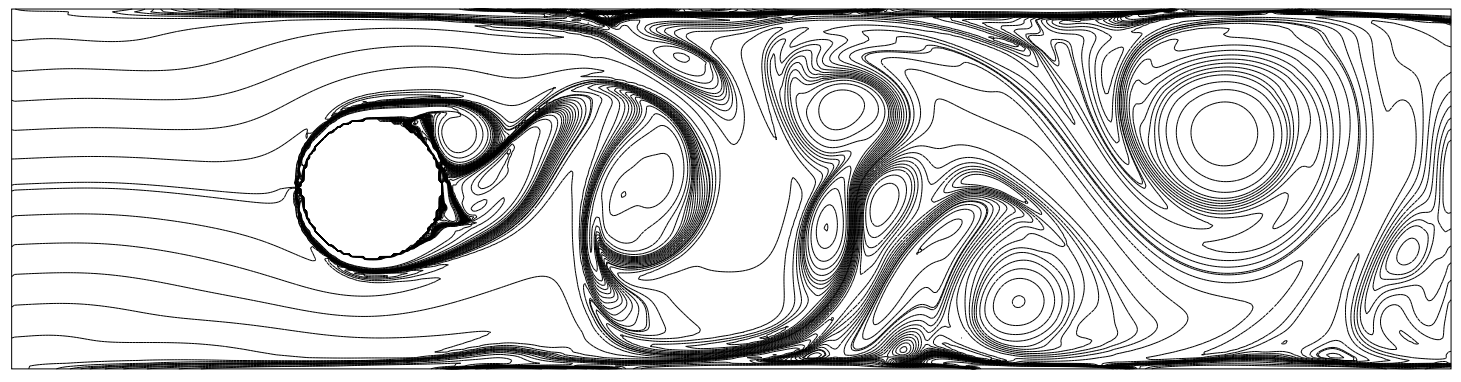

Figure 8: Solution at $R e=10000$

série 2 (1994).

[6] C. Canuto, M.Y. Hussaini, A. Quarteroni, T.A. Zang, Spectral methods in fluid dynamics, Springer-Verlag (1988).

[7] C. Chui, Wavelets : A tutorial in theory and applications, Academic Press (1992).

[8] G. Danabasoglu, S. Biringen, C.L. Streett, Application of the spectral multidomain method to the NavierStokes equations, J. Comp. Phys. 113, $n^{0} 2$ (1994).

[9] S.M. Deshpande, S.S. Desai, R. Narasimha (Eds), Numerical methods in fluid dynamics, Proceedings of the $14^{\text {th }}$ Int. Conf. , Lecture notes in physics 453 (1995).

[10] M. Farge, E. Goiraud, Y. Meyer, F. Pascal, M.V. Wickerhauser, Improved predictability of two-dimensional turbulent flows using wavelet packet compression, Fluid Dyn. Res. 10 (1992).

[11] C.A. Fletcher, Computational techniques for fluid dynamics, Vol.1 \& 2, Springer-Verlag (1991).

[12] V. Girault, P.-A. Raviart, Finite element methods for Navier-Stokes equations : Theory and algorithms, Springer-Verlag (1986).

[13] D. Goldstein, R. Handler, L. Sirovich, Modeling a no-slip flow boundary with an external force field, J. Comp. Phys. 105, $n^{0} 2$ (1993).

[14] P.M. Gresho, Incompressible fluid $d y$ namics : Some fundamental formulation issues, Ann. Rev. Fluid Mech. 23 (1991).

[15] W. Hackbush, Multigrid methods and applications, Springer-Verlag (1985).

[16] M. Hafez (Ed), Computational fluid dynamics, Proceedings of the $6^{\text {th }}$ Int. Symp. (1995).

[17] J.G. Heywood, K. Masuda, R. Rautwann, S.A. Solarnikov (Eds), The Navier-Stokes equations - Theory and numerical methods, Proceedings of the $2^{\text {nd }}$ Conf. , Lecture notes in math. 1530 (1992).

[18] Ch. Hirsch, Numerical Computation of internal and external flows, Vol. 1 \& 2 (1990).

[19] P. Le Quere, T. Alziary de Roquefort, Computation of natural convection in two-dimensional cavities with Chebyshev polynomials, J. Comp. Phys. 57 (1985). 
[20] S. Mallat, Z. Zhang, Matching pursuits with time-frequency dictionaries, Report 619 Courant Institute (1992).

[21] M. Marion, R. Temam, Navier-Stokes equations : Theory and approximation, Handbook of Numerical Analysis (to appear).

[22] Y. Meyer, S. Roques (Eds), Progress in wavelet analysis and applications, Proceedings of the Int. Conf. (1992).

[23] M. Napolitano, F. Sabetta (Eds), Numerical methods in fluid dynamics, Proceedings of the $13^{\text {th }}$ Int. Conf. , Lecture notes in physics 414 (1993).

[24] S. V. Patankar Calculation procedure for viscous incompressible flows in complex geometries, J. Numer. Heat Transfer 14, $n^{0} 3$ (1988).

[25] R. Peyret, T.D. Taylor, Computational methods for fluid flow, SpringerVerlag (1983).

[26] M.B. Ruskai, G. Beylkin, R. Coifman, I. Daubechies, S. Mallat, Y. Meyer, L. Raphael (Eds), Wavelets and their applications, Jones and Barlett Publishers (1992).
[27] E.M. Saiki, S. Biringen, Numerical simulation of a cylinder in uniform flow : Application of a virtual boundary method, J. Comp. Phys. 123 (1996).

[28] R.L. Sani, P.M. Gresho, Résumé and remarks on the open boundary condition minisymposium, Int. J. Num. Meth. in Fluids, 18 (1994).

[29] C. Taylor, J.H. Chin, G.M. Homsy (Eds), Numerical methods in laminar and turbulent flow, Vol.7, Part 1 \& 2, Proceedings of the $7^{\text {th }}$ Int. Conf (1991).

[30] F. Thomasset, Implementation of finite elements methods for NavierStokes equations, Springer-Verlag (1981).

[31] R. Temam, Navier-Stokes equations, North-Holland (1984).

[32] C.H. Williamson, Oblique and parallel modes of vortex shedding in the wake of a circular cylinder at low Reynolds numbers, J. Fluid Mech. 206 (1989).

[33] N.G. Wright, P.H. Gaskell An efficient multigrid approach to solving highly recirculating flows, Comput. Fluids 24, $n^{0} 1$ (1995). 